\title{
The type N Karlhede bound is sharp
}

\author{
R. Milson, N. Pelavas \\ Dept. Mathematics and Statistics, Dalhousie University \\ Halifax NS B3H 3J5, Canada \\ E-mail: rmilson@dal.ca, pelavas@mathstat.dal.ca
}

\begin{abstract}
We present a family of four-dimensional Lorentzian manifolds whose invariant classification requires the seventh covariant derivative of the curvature tensor. The spacetimes in questions are null radiation, type $\mathrm{N}$ solutions on an antide Sitter background. The large order of the bound is due to the fact that these spacetimes are properly $\mathrm{CH}_{2}$, i.e., curvature homogeneous of order 2 but nonhomogeneous. This means that tetrad components of $R, \nabla R, \nabla^{(2)} R$ are constant, and that essential coordinates first appear as components of $\nabla^{(3)} R$. Covariant derivatives of orders 4,5,6 yield one additional invariant each, and $\nabla^{(7)} R$ is needed for invariant classification. Thus, our class proves that the bound of 7 on the order of the covariant derivative, first established by Karlhede, is sharp. Our finding corrects an outstanding assertion that invariant classification of four-dimensional Lorentzian manifolds requires at most $\nabla^{(6)} R$.

PACS numbers: 04.20.Jb, 02.40.Ky
\end{abstract}

AMS classification scheme numbers: $53 \mathrm{C} 50$

The equivalence problem for pseudo-Riemannian geometry is of central importance to general relativity. The invariant classification (IC) of a metric requires knowledge of the curvature tensor and its covariant derivatives $R, \nabla R, \ldots, \nabla^{(q)} R$, up to sufficiently high order. The IC order, the smallest value of $q$ required for invariant classification, depends on the spacetime in question. In general, spacetimes without isometries (Killing vectors) and with an algebraically special Weyl tensor require a larger IC order. An open problem in general relativity is the maximum IC order that can occur in the equivalence problem for four-dimensional, Lorentzian metrics.

Cartan [4] was the first to formulate the equivalence problem in terms of moving frames, structure equations, and differential invariants, and to provide an initial estimate of $q \leq n(n+1) / 2$, where $n$ is the dimension of the underlying manifold $M$. Cartan's ideas were first applied to four-dimensional relativity by Brans 3 . Subsequently, Karlhede introduced a simplified algorithm based on the Petrov and Segre classifications of the curvature tensor [10, which provided better estimates for the IC order. The algorithm was refined and implemented in a computer algebra system by MacCallum, Åman [7, and others; see [13] for a recent review.

Karlhede's counting argument establishes a bound of $q \leq \operatorname{dim} G_{0}+n+1$, where $G_{0}$ is the isotropy group of $R$. Using the well-known Petrov-Penrose classification of the Weyl tensor, the argument yields $q \leq 5$ for Petrov types I, II, III; $q \leq 7$ for Petrov types N, D; and $q \leq 8$ for type Ot. These bounds have been improved by careful analysis. It is now known that $q \leq 6$ for type D [5], and that $q \leq 6$ for type

$\ddagger$ Here, one has to consider the possible symmetries of the Ricci tensor. 
$\mathrm{O}$ [14. However, it is not known whether these type $\mathrm{D}$ and type $\mathrm{O}$ bounds are sharp. Detailed analysis of vacuum type $\mathrm{N}$ solutions yields $q \leq 5$ [9]. A similar analysis of non-vacuum type $\mathrm{N}$ solutions produced a claim of $q \leq 5$ [8]. Subsequently, examples of type $\mathrm{N}$ solutions with $q=5$ were discovered [16].

However, contrary to the above findings, we exhibit an example of a type N, nonvacuum solution that has IC order $q=7$, and thereby show that the type N Karlhede bound is sharp. In our best estimation, the apparent discrepancy between our result and previous claims is due by a subtle error in the analysis of one subcase in [8].

Let us begin by reviewing the Karlhede IC algorithm. Let $(M, g)$ be an $n$ dimensional pseudo-Riemannian manifold. Let $\eta_{a b}$ be a constant, non-degenerate quadratic form having the same signature as the metric $g$. Henceforth, we use $\eta_{a b}$ to raise and lower frame indices, which we denote by $a, b, c=1, \ldots, n$, and say that a coframe $\boldsymbol{\theta}^{a}$ is $\eta$-orthogonal if $g=\eta_{a b} \boldsymbol{\theta}^{a} \boldsymbol{\theta}^{b}$. Let $O(\eta)=\left\{\left(X_{b}^{a}\right): X^{a}{ }_{c} \eta_{a b} X^{b}{ }_{d}=\eta_{c d}\right\}$ denote the group of $\eta$-orthogonal transformations. Let $\mathfrak{o}(\eta)=\left\{\left(A_{b}^{a}\right): A_{(a b)}=0\right\}$ denote the $n(n-1) / 2$-dimensional Lie algebra of $\eta$ skew-symmetric transformations.

1. Let $q=0, G_{-1}=O(\eta), t_{-1}=0$. All $\eta$-orthogonal frames are permitted.

2. Compute $\nabla^{(q)} R$ relative to a permitted $\eta$-orthogonal frame.

3. Determine $G_{q} \subset G_{q-1}$, the isotropy group of $\nabla^{(q)} R$.

4. Restrict the frame freedom to $G_{q}$ by putting $\nabla^{(q)} R$ into standard form (normalizing some components to a constant.)

5. The functions in the set $R^{q}=\left\{R_{a b c d}, R_{a b c d ; e_{1}}, \ldots, R_{a b c d ; e_{1} \ldots, e_{q}}\right\}$ are now differential invariants. Find $t_{q}$, the number of independent functions over $M$ in $R^{q}$.

6. If $\operatorname{dim} G_{q}<\operatorname{dim} G_{q-1}$ or $t_{q}>t_{q-1}$, then increase $q$ by one, and go to step 2 .

7. Otherwise, the algorithm terminates. The differential invariants in $R^{q-1}$ furnish essential coordinates. The isometry group has dimension $n-t_{q}+\operatorname{dim} G_{q}$. The orbits have dimension $n-t_{q}$.

In principle, the essential coordinates obtained via the algorithm allow the metric to be expressed in a canonical form that incorporates the other differential invariants as essential constants and essential functional parameters.

An analysis of the algorithm reveals that the conditions for an IC order of $q=7$ are very stringent. In [5], Collins and d'Inverno list the following necessary conditions:

(C1) The components of the curvature tensor must be constants.

(C2) The invariance group at zeroth order $G_{0}$, must have dimension 2.

(C3) The dimension of the invariance group and the number of functionally independent components must not both change on differentiating.

(C4) We must produce at most one new functionally independent component on differentiating.

(C5) The dimension of the invariance group must go down by at most one dimension on differentiating.

Therefore, the search for a metric with $q=7$ must focus on a very particular class of geometries.

To that end, let us say that a pseudo-Riemannian manifold is curvature homogeneous of order $k$, or $\mathrm{CH}_{k}$ for short, if the components of the curvature tensor and its first $k$ covariant derivatives are constant relative to an $\eta$-orthogonal frame. Let 
us also say that $M$ is properly $\mathrm{CH}_{k}$ if it belongs to class $\mathrm{CH}_{k}$, but is not a (locally) homogeneous space 2. Proper $\mathrm{CH}$ metrics are of central importance in invariant classification, because they are the natural candidates for obtaining sharp bounds on the IC order.

A homogeneous space can be characterized as a geometry without any essential coordinates. In other words, $t_{q}=0$; all differential invariants are essential constants that define the structure of the corresponding Lie algebra. Thus, as a special case of the Karlhede algorithm, we have the following result, first proved by Singer [15] for Riemannian manifolds.

Theorem 1 If $(M, g)$ is a $\mathrm{CH}_{k+1}$ manifold and $\operatorname{dim} G_{k}=\operatorname{dim} G_{k+1}$, then $M$ is a (locally) homogeneous space.

Consequently, in a proper $\mathrm{CH}_{k}$ manifold, the first differential invariant arises only at order $k+1$. Thus, if only 1 new differential invariant arises at each subsequent order, a proper $n$-dimensional $\mathrm{CH}_{k}$ manifold could, in principle, have an IC order $q=k+n+1$. Below, we prove that this possibility can be realized with $n=4$ and $k=2$.

We plan to report on a classification of proper $\mathrm{CH}_{2}$ and $\mathrm{CH}_{1}$ four-dimensional, Lorentzian geometries in a subsequent publication [11. In principle, this will allow us to investigate the sharpness of the IC bound for type D and O solutions, as well. In this letter, we focus on type $\mathrm{N}$, and exhibit a family of type $\mathrm{N}$ metrics that realize the $q=7$ bound. The spacetimes in questions belong to the class of null radiation, type $\mathrm{N}$ solutions on an anti-de Sitter background. This general class of exact solutions was first investigated in [6] and [12, but it was not known that a $\mathrm{CH}_{2}$ geometry could arise as a special case.

Henceforth, $n=4$ and all tetrads $\left\{\boldsymbol{e}_{a}\right\}=(\boldsymbol{m}, \overline{\boldsymbol{m}}, \boldsymbol{n}, \boldsymbol{\ell})$ are complex, nullorthogonal with

$$
\boldsymbol{\theta}_{1}=\boldsymbol{\theta}^{2}, \boldsymbol{\theta}_{3}=-\boldsymbol{\theta}^{4}, \quad \boldsymbol{\theta}^{2}=\overline{\boldsymbol{\theta}}^{1}, \boldsymbol{\theta}^{3}=\overline{\boldsymbol{\theta}}^{3}, \boldsymbol{\theta}^{4}=\overline{\boldsymbol{\theta}}^{4}
$$

denoting the dual coframe. The metric is given by

$$
g=2 \boldsymbol{\theta}^{1} \boldsymbol{\theta}^{2}-2 \boldsymbol{\theta}^{3} \boldsymbol{\theta}^{4} .
$$

The connection 1-form and the the curvature 2 -form are defined, respectively by

$$
\begin{aligned}
& d \boldsymbol{\theta}^{a}=\boldsymbol{\omega}_{b}^{a} \wedge \boldsymbol{\theta}^{b}, \quad \boldsymbol{\omega}_{(a b)}=0 \\
& \boldsymbol{\Omega}^{a}{ }_{b}=d \boldsymbol{\omega}^{a}{ }_{b}+\boldsymbol{\omega}^{a}{ }_{c} \wedge \boldsymbol{\omega}^{c}{ }_{d} .
\end{aligned}
$$

The connection components are labeled by the 12 Newman-Penrose scalars:

$$
\begin{gathered}
-\boldsymbol{\omega}_{14}=\sigma \boldsymbol{\theta}^{1}+\rho \boldsymbol{\theta}^{2}+\tau \boldsymbol{\theta}^{3}+\kappa \boldsymbol{\theta}^{4} ; \\
\boldsymbol{\omega}_{23}=\mu \boldsymbol{\theta}^{1}+\lambda \boldsymbol{\theta}^{2}+\nu \boldsymbol{\theta}^{3}+\pi \boldsymbol{\theta}^{4} ; \\
-\left(\boldsymbol{\omega}_{12}+\boldsymbol{\omega}_{34}\right) / 2=\beta \boldsymbol{\theta}^{1}+\alpha \boldsymbol{\theta}^{2}+\gamma \boldsymbol{\theta}^{3}+\epsilon \boldsymbol{\theta}^{4} .
\end{gathered}
$$

The curvature components are labelled by the Ricci scalar $\Lambda=\bar{\Lambda}$, traceless Ricci components $\Phi_{A B}=\bar{\Phi}_{B A}, A, B=0,1,2$, and Weyl components $\Psi_{C}, C=0, \ldots, 4$ :

$$
\begin{gathered}
\boldsymbol{\Omega}_{14}=\Phi_{01}\left(\boldsymbol{\theta}^{34}-\boldsymbol{\theta}^{12}\right)-\Phi_{02} \boldsymbol{\theta}^{13}+\Phi_{00} \boldsymbol{\theta}^{24}+\Psi_{0} \boldsymbol{\theta}^{14}-\left(\Psi_{2}+2 \Lambda\right) \boldsymbol{\theta}^{23}+\Psi_{1}\left(\boldsymbol{\theta}^{12}+\boldsymbol{\theta}^{34}\right) \\
\boldsymbol{\Omega}_{23}=\Phi_{21}\left(\boldsymbol{\theta}^{12}-\boldsymbol{\theta}^{34}\right)+\Phi_{22} \boldsymbol{\theta}^{13}-\Phi_{20} \boldsymbol{\theta}^{24}+\Psi_{4} \boldsymbol{\theta}^{23}-\left(\Psi_{2}+2 \Lambda\right) \boldsymbol{\theta}^{14}-\Psi_{3}\left(\boldsymbol{\theta}^{12}+\boldsymbol{\theta}^{34}\right) \\
\left(\boldsymbol{\Omega}_{12}+\boldsymbol{\Omega}_{34}\right) / 2=-\Phi_{12} \boldsymbol{\theta}^{13}+\Phi_{10} \boldsymbol{\theta}^{24}+\Psi_{1} \boldsymbol{\theta}^{14}-\Psi_{3} \boldsymbol{\theta}^{23}+ \\
+\Phi_{11}\left(\boldsymbol{\theta}^{34}-\boldsymbol{\theta}^{12}\right)+\left(\Psi_{2}-\Lambda\right)\left(\boldsymbol{\theta}^{12}+\boldsymbol{\theta}^{34}\right),
\end{gathered}
$$


where $\boldsymbol{\theta}^{a b}=\boldsymbol{\theta}^{a} \wedge \boldsymbol{\theta}^{b}$. Let $\boldsymbol{A}_{\alpha}=\left(A_{b \alpha}^{a}\right)_{a, b=1}^{n}, \alpha=1, \ldots, n(n-1) / 2$ be a basis of $\mathfrak{o}(\eta)$ with $C^{\alpha}{ }_{\beta \gamma}$ the corresponding structure constants. Let $\Gamma_{a}^{\alpha}, R_{a b}^{\alpha}$, where

$$
\begin{aligned}
\boldsymbol{\omega}_{b}^{a} & =A_{b \alpha}^{a} \Gamma_{c}^{\alpha} \boldsymbol{\theta}^{c}, \\
\boldsymbol{\Omega}_{b}^{a} & =\frac{1}{2} A_{b \alpha}^{a} R^{\alpha}{ }_{c d} \boldsymbol{\theta}^{c d},
\end{aligned}
$$

denote the connection and curvature components relative to the basis.

Let $\tilde{\Lambda}<0$ be a negative constant. Following [12, a class of exact solutions for coupled electromagnetic radiation and gravity propagating in a negatively curved background is given by

$g_{i j} d x^{i} d x^{j}=2 p^{-2} d \zeta d \bar{\zeta}-2 q^{2} p^{-2}\left(\left(-\left(\tilde{\Lambda} A^{2}+B \bar{B}\right) r^{2}+r q_{s} / q+2 H p / q\right) d s+d r\right) d s$, (11) where $\zeta, \bar{\zeta}, r, s$ are coordinates, where

$$
\begin{aligned}
& p=1+\tilde{\Lambda} \zeta \bar{\zeta} \\
& q=(1-\tilde{\Lambda} \zeta \bar{\zeta}) A+\bar{B} \zeta+B \bar{\zeta} \\
& A=A(s), B=B(s), A=\bar{A} \\
& H=H(\zeta, \bar{\zeta}, s), \quad H_{\zeta \bar{\zeta}}+2 \tilde{\Lambda} p^{-2} H=f \bar{f} p / q,
\end{aligned}
$$

and where

$$
f d \zeta \wedge d s+\bar{f} d \bar{\zeta} \wedge d s, \quad f=f(\zeta, s)
$$

is the electromagnetic field.

Let $\tilde{\tau}_{1} \neq 0, \tilde{\mu}_{2} \neq 8 / 5$ be real constants, and let $F(s)>0$ be a positive function of one variable. Let us also set

$$
F_{1}(s)=F^{\prime}(s) / F(s), \quad F_{2}(s)=\left(F_{1}^{\prime}(s)-F_{1}^{2}(s) / 8\right) / \sqrt{F(s)},
$$

and demand that $F_{2}^{\prime}(s) \neq 0$. We perform a change of coordinates and a specialization of the parameters in (11) as follows:

$$
\begin{aligned}
& \tilde{\Lambda}=-\tilde{\tau}_{1}^{2}, \quad A=1, \quad B=-e^{3 i s} \tilde{\tau}_{1}, \\
& \left.H=\left[36-72 / p+\left(27+16 \tilde{\tau}_{1}^{2} F(s)\right) q / p+\left(10 \tilde{\mu}_{2}-16\right) p^{3} / q^{3}\right)\right] /\left(32 \tilde{\tau}_{1}^{2}\right) \\
& a=\left(\tilde{\tau}_{1} / p\right) \Im\left(e^{-3 i s} \zeta\right) \\
& b=\log (p)-\log (q) \\
& t=r+a e^{b}\left(3 / 2+e^{2 b}\left(1+4 a^{2} / 3\right)\right) / \tilde{\tau}_{1}^{2} .
\end{aligned}
$$

The resulting metric can be expressed in terms of real coordinates $a, b, s, t$ and a null-orthogonal coframe as follows:

$$
\begin{aligned}
\boldsymbol{\theta}^{1} & =\left(d b / 2+i(a d b+d a)-e^{b}\left(a+i\left(a^{2}+\tilde{\mu}_{2} / 2-5 / 4\right)\right) d s\right) / \tilde{\tau}_{1}, \\
\boldsymbol{\theta}^{2} & =\left(d b / 2-i(a d b+d a)-e^{b}\left(a-i\left(a^{2}+\tilde{\mu}_{2} / 2-5 / 4\right)\right) d s\right) / \tilde{\tau}_{1}, \\
\boldsymbol{\theta}^{3} & =e^{b} d s \\
\boldsymbol{\theta}^{4} & =e^{-3 b} d t-\left(\tilde{\mu}_{2} / \tilde{\tau}_{1}^{2}\right)(d a+a d b) \\
& \quad+\left(F(s) e^{-3 b}-6 a e^{-2 b} t+\left(\tilde{\mu}_{2} / \tilde{\tau}_{1}^{2}\right)\left(a^{2}+\tilde{\mu}_{2} / 4-5 / 8\right) e^{b}\right) d s .
\end{aligned}
$$

Using (3)-(8) to calculate the connection and curvature yields

$$
\begin{aligned}
& \Lambda=-\tilde{\tau}_{1}^{2}, \\
& \Phi_{22}=\Psi_{4} / 3=-4+5 \tilde{\mu}_{2} / 2, \\
& \tau=-\pi=2 \beta=2 \alpha / 3=\tilde{\tau}_{1}, \\
& \gamma=3 i / 2 \\
& \mu=\lambda+2 i=i \tilde{\mu}_{2}, \\
& \nu=-3 i \tilde{\tau}_{1} e^{-3 b} t
\end{aligned}
$$


with all other NP scalars equal to zero.

As a basis of $\mathfrak{o}(\eta)$ let us take

$$
\left(\boldsymbol{A}_{\alpha}\right)=\left(\boldsymbol{e}_{23}, \boldsymbol{e}_{13}, \boldsymbol{e}_{34}, \boldsymbol{e}_{12}, \boldsymbol{e}_{14}+\boldsymbol{e}_{24}, \boldsymbol{e}_{14}-\boldsymbol{e}_{24}\right) .
$$

where $\boldsymbol{e}_{a b}=\boldsymbol{e}_{a} \wedge \boldsymbol{e}_{b}$ is a basic bivector. Note that, because $\tilde{\mu}_{2} \neq 8 / 5$, we have $\Phi_{22}, \Psi_{4} \neq 0$, and hence the null rotations $\boldsymbol{A}_{5}, \boldsymbol{A}_{6}$ generate $G_{0}$. Since the curvature components are constant, we have

$$
\nabla_{c} R_{a b}^{\alpha}=\sum_{\beta=1}^{4}\left(\boldsymbol{A}_{\beta} \cdot R\right)^{\alpha}{ }_{a b} \Gamma_{c}^{\beta},
$$

where

$$
\left(\boldsymbol{A}_{\beta} \cdot R\right)^{\alpha}{ }_{a b}=C^{\alpha}{ }_{\beta \gamma} R^{\gamma}{ }_{a b}+2 R^{\alpha}{ }_{c[a} A^{c}{ }_{b] \beta}
$$

denotes the standard action of $\mathfrak{o}(\eta)$ on the vector space of curvature-type tensors. Setting

$$
\Gamma^{(1)}=\left(\begin{array}{cccc}
\sigma & \rho & \tau & \kappa \\
\bar{\rho} & \bar{\sigma} & \bar{\tau} & \bar{\kappa} \\
-\beta-\bar{\alpha} & -\alpha-\bar{\beta} & -2 \gamma_{1} & -2 \epsilon_{1} \\
-\beta+\bar{\alpha} & -\alpha+\bar{\beta} & -2 i \gamma_{2} & -2 i \epsilon_{2}
\end{array}\right)
$$

where a subscript of 1 and 2 on a spin coefficient denotes the real and imaginary part, respectively, lets us express the covariant derivative, symbolically, as

$$
\nabla R=\Gamma^{(1)} \cdot R .
$$

Since the components of $\Gamma^{(1)}$ are all constant, it follows that the metric is $\mathrm{CH}_{1}$. Next, since $\tilde{\tau}_{1} \neq 0$, we have that $\Gamma^{(1)}$, and thereby $\nabla R$, are invariant with respect $\boldsymbol{A}_{6}$. Thus, $G_{1}$ is generated by $\boldsymbol{A}_{6}$. Setting

$$
\Gamma^{(2)}=\left(\begin{array}{cccc}
\Gamma^{(1)} & & \\
\frac{1}{2}(\mu+\bar{\lambda}) & \frac{1}{2}(\lambda+\bar{\mu}) & \nu_{1} & \pi_{1}
\end{array}\right)
$$

and writing

$$
\nabla^{(2)} R=\Gamma^{(2)} \cdot \nabla R,
$$

we infer that the metric is $\mathrm{CH}_{2}$. Note that $\Gamma^{(2)}$, and thereby $\nabla^{(2)} R$ are not left invariant by $\boldsymbol{A}_{6}$. Hence, $G_{2}$ is trivial, and

$$
\nabla^{(3)} R=\Gamma^{(3)} \cdot \nabla^{(2)} R
$$

where

$$
\Gamma^{(3)}=\left(\begin{array}{cccc} 
& \Gamma^{(2)} & & \\
\frac{1}{2}(\bar{\lambda}-\mu) & \frac{1}{2}(\bar{\mu}-\lambda) & \nu_{2} & \pi_{2}
\end{array}\right) .
$$

Since $\nu_{2}$ is not a constant, the metric is a proper $\mathrm{CH}_{2}$ (not a $\mathrm{CH}_{3}$ ). Indeed, arising as a component of $\nabla^{(3)} R$,

$$
I_{1}:=\nu_{2} /\left(3 \tilde{\tau}_{1}\right)=e^{-3 b} t
$$


is our first non-constant differential invariant. To calculate the frame derivatives, let us introduce the following real coframe:

$$
\begin{aligned}
\tilde{\boldsymbol{\theta}}^{1} & =\tilde{\tau}_{1} / 2\left(\boldsymbol{\theta}^{1}+\boldsymbol{\theta}^{2}\right) \\
& =d b / 2-a e^{b} d s, \\
\tilde{\boldsymbol{\theta}}^{2} & =-i \tilde{\tau}_{1} / 2\left(\boldsymbol{\theta}^{1}-\boldsymbol{\theta}^{2}\right)+\left(2 \tilde{\mu}_{2}-5\right) / 4 \boldsymbol{\theta}^{3} \\
& =d a+a d b-a^{2} e^{b} d s, \\
\tilde{\boldsymbol{\theta}}^{3} & =\boldsymbol{\theta}^{3}=e^{b} d s \\
\tilde{\boldsymbol{\theta}}^{4} & =\left(\tilde{\mu}_{2} / \tilde{\tau}_{1}\right)\left(-i / 2\left(\boldsymbol{\theta}^{1}-\boldsymbol{\theta}^{2}\right)+\left(2 \tilde{\mu}_{2}-5\right) /\left(8 \tilde{\tau}_{1}\right) \boldsymbol{\theta}^{3}\right)+\boldsymbol{\theta}^{4} \\
& =e^{-3 b}\left(d t+\left(F(s)-6 a e^{b} t\right) d s\right)
\end{aligned}
$$

Even though this coframe is not null-orthogonal, it's form simplifies the derivations that follow. Since the two coframes are related by a constant linear transformation, they yield the same differential invariants.

Continuing, we have

$$
d I_{1}=-6 I_{1} \tilde{\boldsymbol{\theta}}^{1}-e^{I_{2}} \tilde{\boldsymbol{\theta}}^{3}+\tilde{\boldsymbol{\theta}}^{4},
$$

where

$$
I_{2}:=\log F(s)-4 b
$$

is our second differential invariant. From the symbolic expression

$$
\nabla^{(4)} R=D \nabla^{(3)} R+\Gamma^{(3)} \cdot \nabla^{(3)} R
$$

we infer that $I_{2}$ can be recovered from the components of $\nabla^{(4)} R$, and that no other functionally independent invariants occur in $\nabla^{(4)} R$. Next,

$$
d I_{2}=-8 \tilde{\boldsymbol{\theta}}^{1}+I_{3} \tilde{\boldsymbol{\theta}}^{3}
$$

where

$$
I_{3}:=e^{-b} F_{1}(s)-8 a
$$

and

$$
d I_{3}=-2 I_{3} \tilde{\boldsymbol{\theta}}^{1}-8 \tilde{\boldsymbol{\theta}}^{2}+\left(I_{3}^{2} / 8+e^{I_{2} / 2} I_{4}\right) \tilde{\boldsymbol{\theta}}^{3}
$$

where

$$
I_{4}:=F_{2}(s) .
$$

Hence, for analogous reasons we obtain $I_{3}$ from $\nabla^{(5)} R$ and $I_{4}$ from $\nabla^{(6)} R$. By assumption, $F_{2}(s)$ is non-constant, and hence $I_{1}, I_{2}, I_{3}, I_{4}$ are functionally independent essential coordinates. Since $n=4$, no functionally independent differential invariants arise in $\nabla^{(7)} R$. Indeed,

$$
d I_{4}=F_{2}^{\prime}(s) d s=e^{-b} F_{2}^{\prime}(s) \tilde{\boldsymbol{\theta}}^{3}=e^{I_{2} / 4} I_{5} \tilde{\boldsymbol{\theta}}^{3},
$$

where

$$
I_{5}:=F_{2}^{\prime}(s)(F(s))^{-1 / 4} .
$$

Since both $I_{4}$ and $I_{5}$ are functions of $s$, and since $F_{2}^{\prime}(s) \neq 0$, locally

$$
I_{5}=\phi\left(I_{4}\right)
$$


where $\phi(x)$ is a function of 1 variable. Therefore, the metric is fully classified by 2 essential constants $\tilde{\tau}_{1}, \tilde{\mu}_{2}$ and by 1 essential functional parameter $\phi(x)$. The above IC process can be summarized as follows:

$$
\left(t_{0}, t_{1}, t_{2}, t_{3}, t_{4}, t_{5}, t_{6}, t_{7}\right)=(0,0,0,1,2,3,4,4) .
$$

It is also possible to obtain the above count of differential invariants directly, by means of a CAS package such as CLASSI 11. Therefore, the metrics described by (22)-(25) have IC order $q=7$. This proves that the type N Karlhede bound is sharp.

\section{Acknowledgments}

We gratefully acknowledge conversations with A. Coley, S. Hervik, and G. Papadopoulos. We also thank J. Åman for useful comments. The research of RM is supported in part by NSERC grant RGPIN-228057-2004.

\section{References}

[1] Åman J E 2007 personal communication

[2] Boeckx E, Kowalski O and Vanhecke L 1996, Riemannian manifolds of conullity two, (River Edge, NJ:World Scientific)

[3] Brans C H 1965 Invariant approach to the geometry of spaces in general relativity J. Math. Phys. 6 95-102

[4] Cartan E 1946 Leçons sur la Géométrie des Espaces de Riemann (Paris: Gauthier-Villars)

[5] Collins J M and d'Inverno R A 1993 The Karlhede classification of type-D non-vacuum spacetimes Class. Quantum Grav. $10343-51$

[6] Díaz A G and Plebański J F 1981 All nontwisting N's with cosmological constant J. Math. Phys. $2655-58$

[7] MacCallum M A H and Åman J E 1986 Algebraically independent nth derivatives of the Riemann curvature spinor in a general spacetime Class. Quantum Grav. 3 1133-41

[8] Machado Ramos M P 1998 Invariant differential operators and the Karlhede classification of type N non-vacuum solutions Class. Quantum Grav. 15 435-54

[9] Machado Ramos M P and Vickers J A G 1996 Invariant differential operators and the Karlhede classification of type N vacuum solutions Class. Quantum Grav. 13 1589-99

[10] Karlhede A 1980 A review of the geometrical equivalence of metrics in general relativity Gen. Rel. Grav. 12 693-707

[11] Milson R and Pelavas N 2007 The curvature homogeneity bound for Lorentzian four-manifolds Preprint

[12] Ozsváth I, Robinson I and Rózga K 1985 Plane-fronted gravitational and electromagnetic waves in spaces with cosmological constant J. Math. Phys 1755-61

[13] Pollney D, Skea J E F and d'Inverno R A 2000 Classifying geometries in general relativity: I. Standard forms for symmetric spinors Class. Quantum Grav. 17 643-63

[14] Paiva F M and Skea J E F 2000 On the invariant classification of conformally flat spacetimes IF/UERJ preprint 2000.002

[15] Singer I M 1960 Infinitesimally homogeneous spaces Comm. Pure Appl. Math. 13 685-97.

[16] Skea J F 2000 A spacetime whose invariant classification requires the fifth covariant derivative of the Riemann tensor Class. Quantum Grav. 17 L69-L74 